\title{
Perfil dos usuários e dos atendimentos na especialidade de Endodontia do CEO-UFRGS entre 2016 e 2017
}

\author{
Valéria Rozales Ehlert*; Simone Bonato Luisi**
}

* Graduada em Odontologia, Universidade Federal do Rio Grande do Sul

** Professora Associada da Faculdade de Odontologia, Universidade Federal do Rio Grande do Sul

Recebido em 06/09/2018. Aprovado em 21/05/2019.

\begin{abstract}
RESUMO
Os Centros de Especialidades Odontológicas (CEO) são uma estratégia da Política Nacional de Saúde Bucal para garantir a atenção secundária em Odontologia, constituindo-se em unidades de referência para a atenção primária. Este estudo transversal analisou as características dos usuários e dos atendimentos na Especialidade de Endodontia do CEO da Universidade Federal do Rio Grande do Sul. Foi formulado um questionário baseado no Manual de Especialidades em Saúde Bucal, acrescido de perguntas que caracterizavam o perfil do usuário e aplicado aos estagiários atuantes no CEO a cada tratamento endodôntico concluído. A amostra totalizou 48 pacientes, obtida por conveniência, em amostragem consecutiva do tipo censo onde foram concluídos 56 tratamentos endodônticos, durante o período de agosto de 2016 a julho de 2017. O perfil dos usuários encaminhados foi, predominantemente, do sexo feminino $(62,50 \%)$, na faixa etária de 31 a 50 anos $(41,67 \%)$ e da raça branca $(64,58 \%)$; os dentes que mais necessitaram tratamento foram os molares $(67,86 \%)$; e na maioria dos casos encontravam-se em condições compatíveis com as preconizadas pelo Manual de Especialidades em Saúde Bucal; os tratamentos foram concluídos em 2 ou 3 consultas $(66,67 \%)$; o tempo de espera dos usuários para atendimento foi inferior a 6 meses $(83,33 \%)$. Os pacientes foram encaminhados por meio da ficha de referência (93,75\%). O estudo evidenciou dados que podem contribuir para o desenvolvimento de estratégias que ampliem a produtividade por meio de investimentos em novas tecnologias e equipamentos que possam reduzir o número de consultas necessárias para a finalização dos tratamentos endodônticos, proporcionando atendimento para um número maior pacientes e reduzindo o tempo de espera.
\end{abstract}

Descritores: Atenção Secundária à Saúde. Especialidades Odontológicas. Endodontia. 


\section{INTRODUÇÃO}

Os serviços de saúde bucal no Brasil, historicamente, caracterizavam-se por ações de baixa complexidade, curativas e mutiladoras, com acesso bastante restrito, principalmente, à faixa etária escolar. Os adultos e os idosos tinham acesso apenas a serviços de urgência, geralmente mutiladores, o que caracterizava a Odontologia como uma das áreas da saúde com maior exclusão social ${ }^{15}$.

Em 2004, o Ministério da Saúde lançou as diretrizes da Política Nacional de Saúde Bucal (PNSB), também conhecida como Programa Brasil Sorridente, que enfatizava a necessidade de se aumentar o acesso ao cuidado da saúde bucal, com visão integral do processo de saúde e doença e incorporando procedimentos e serviços de média e alta complexidade ${ }^{9}$.

No âmbito da assistência, a PNSB apresenta diretrizes que apontam, fundamentalmente, para a ampliação e a qualificação da Atenção Básica (AB) em saúde bucal, por meio da realização de atividades de promoção, prevenção, assistência e reabilitação, possibilitando o acesso a todas as faixas etárias, promovendo atendimento na atenção secundária, por meio da implantação dos Centros de Especialidades Odontológicas (CEO), que desenvolvem o papel de assegurar a retaguarda da atenção básica, aumentando a resolutividade da mesma ${ }^{4}$. Tais serviços devem se constituir em unidades de referência para a atenção primária, integrados ao processo de planejamento locorregional, ofertando as especialidades de periodontia, endodontia, atendimento a pacientes com necessidades especiais, diagnóstico bucal com ênfase na detecção do câncer de boca e cirurgia oral menor ${ }^{1}$.

Em 2006 foi celebrado um convênio entre a Universidade Federal do Rio Grande do Sul (UFRGS), o Ministério da Saúde e a Prefeitura Municipal de Porto Alegre para a implantação do
CEO na UFRGS. O objetivo foi integrar a rede de atenção especializada em Odontologia da Secretaria Municipal de Saúde de Porto Alegre, com a perspectiva de formar profissionais capacitados e qualificados para atuarem nesses serviços públicos especializados em saúde e assumir uma posição colaborativa e estratégica no desempenho da UFRGS junto ao SUS ${ }^{22}$.

Em 2014 foi instituído o componente GraduaCEO - Brasil Sorridente, no âmbito da PNSB, que compõe a Rede de Atenção à Saúde (RAS), desenvolvido numa parceria entre Ministério da Saúde e da Educação. Um dos objetivos é integrar as clínicas odontológicas das Instituições de Ensino Superior (IES) à rede pública na busca de garantir a universalidade de acesso, equidade e integralidade na atenção à saúde bucal ${ }^{3}$.

Em relação à especialidade de Endodontia, a responsabilidade do nível da atenção secundária é realizar tratamento endodôntico em dentes com polpa viva, com polpa sem vitalidade, retratamento endodôntico, tratamento de perfurações radiculares e após o tratamento concluído é necessário contrarreferenciar o paciente à Unidade Básica de Saúde (UBS). O usuário encaminhado ao CEO para a especialidade de Endodontia deve apresentar algumas condições para ser atendido na atenção especializada. Com relação ao dente, as condições são remoção total do tecido cariado, colocação de curativo de demora e material restaurador provisório, o dente deve estar com coroa clínica suficiente para colocação dos grampos de isolamento absoluto e deve estar sem mobilidade acentuada e com menos de $2 / 3$ de extrusão por perda do antagonista. Com relação à cavidade bucal, o usuário deve apresentar adequação do meio bucal com remoção dos focos infecciosos ${ }^{2}$.

Estudos sobre atenção secundária em saúde bucal estão em ascensão no Brasil, sendo 
importantes para a avaliação destas ações em virtude da complexidade organizacional dos serviços $^{25}$. Somando esse fato à grande demanda por tratamento odontológico especializado na rede pública de saúde do Brasil, o presente estudo descreveu o perfil dos usuários e dos atendimentos realizados na especialidade de endodontia no CEO da UFRGS a fim de analisar as características dos serviços ofertados na atenção secundária e fornecer informações que possam impulsionar melhorias no serviço analisado.

\section{METODOLOGIA}

O presente estudo do tipo transversal com análise descritiva do conjunto de dados foi aprovado pelo Comitê de Ética em Pesquisa da UFRGS, conforme CAEE $\mathrm{n}^{\mathrm{o}} 38025514$. 3.0000.5347. Realizado no ambulatório de atendimento do CEO, na área de Endodontia da UFRGS, por meio da análise das respostas a um questionário aplicado aos estagiários, referente a cada tratamento concluído na especialidade de Endodontia. O questionário foi formulado com base no Manual de Especialidades em Saúde $\mathrm{Bucal}^{8}$, acrescido de perguntas que caracterizavam o perfil do usuário e foi entregue impresso ao estagiário, juntamente com o prontuário.

A população estudada foi obtida por conveniência, em amostragem consecutiva do tipo censo, sendo incluídos no estudo todos os questionários respondidos pelos estagiários do CEO durante o período de agosto de 2016 a julho de 2017. Os dados são referentes aos atendimentos prestados aos usuários na especialidade de Endodontia do CEO da UFRGS, referenciados pela $\mathrm{AB}$.

Os estagiários que aceitaram participar da pesquisa assinaram o Termo de Consentimento Livre e Esclarecido elaborado a partir da resolução número 466/12 do Conselho Nacional de Saúde. Os critérios de inclusão para participação no estudo foram: participação voluntária e anônima na pesquisa, leitura e assinatura do termo de consentimento livre e esclarecido; estar atuando no CEO de Endodontia da UFRGS como acadêmico ou como cirurgião-dentista integrante da Residência Integrada em Saúde Bucal com ênfase em Endodontia.

A análise das respostas coletadas foi realizada por meio de estatísticas descritivas.

\section{RESULTADOS}

Quarenta e oito pacientes receberam tratamento endodôntico no período avaliado. Destes, 30 eram do sexo feminino (62,5\%), 16 do sexo masculino $(33,33 \%)$ e 2 pacientes não informaram o sexo $(4,17 \%)$. A faixa etária predominante foi de 31 a 50 anos, somando $41,67 \%$ dos casos, 39,58\% possuíam 16 a 30 anos, $16,67 \%$ possuíam 51 a 80 anos e 2,08\% possuíam 12 a 15 anos. A maior parte dos pacientes atendidos eram de etnia branca (64,58\%), 18,75\% eram negros e 16,67\% pardos. $\mathrm{Na}$ tabela 1 encontram-se as Unidades de Saúde e as Gerências Distritais de proveniência dos pacientes.

Participaram dessa pesquisa estagiários da graduação do sétimo, oitavo e décimo semestres, os quais juntos atenderam 31 pacientes $(64,58 \%)$ e alunos de pós-graduação, os quais atenderam 17 pacientes $(35,42 \%)$. Foram concluídos 56 tratamentos endodônticos durante o período estudado. Os molares (18 superiores e 20 inferiores) representaram $67,86 \%$ dos casos concluídos, seguidos dos pré-molares (16,08\%), incisivos $(7,15 \%)$ e caninos $(5,37 \%)$. Na tabela 2 encontram-se dados referentes ao número de consultas utilizadas para a conclusão dos tratamentos endodônticos, constatando que a maior parte dos casos foram concluídos em 2 ou 3 consultas. 
Tabela 1. Distribuição dos pacientes de acordo com a Gerência Distrital e a Unidade de Saúde de proveniência

\begin{tabular}{|c|c|c|c|}
\hline Gerência Distrital & Unidade de Saúde & $\mathbf{N}^{0}$ pacientes & $\%$ \\
\hline \multirow{3}{*}{ Glória/Cruzeiro/Cristal (GCC) } & Alto Embratel & 1 & $2,08 \%$ \\
\hline & Belém Velho & 1 & $2,08 \%$ \\
\hline & Divisa & 1 & $2,08 \%$ \\
\hline \multirow{6}{*}{ Leste/Noroeste (LENO) } & Bom Jesus & 5 & $10,42 \%$ \\
\hline & Divina Providência & 1 & $2,08 \%$ \\
\hline & Jardim FAPA & 1 & $2,08 \%$ \\
\hline & Timbaúva & 1 & $2,08 \%$ \\
\hline & Vila Jardim & 1 & $2,08 \%$ \\
\hline & Vila Pinto & 1 & $2,08 \%$ \\
\hline \multirow{8}{*}{ Sul/Centro-Sul (SCS) } & Calábria & 1 & $2,08 \%$ \\
\hline & Campo Novo & 1 & $2,08 \%$ \\
\hline & Cidade de Deus & 1 & $2,08 \%$ \\
\hline & Guarujá & 1 & $2,08 \%$ \\
\hline & Moradas da Hípica & 1 & $2,08 \%$ \\
\hline & São Vicente Mártir & 1 & $2,08 \%$ \\
\hline & Tristeza & 1 & $2,08 \%$ \\
\hline & Vila Nova Ipanema & 1 & $2,08 \%$ \\
\hline \multirow{6}{*}{$\begin{array}{c}\text { Noroeste/Humaitá/ } \\
\text { Navegantes/Ilhas (NHNI) }\end{array}$} & Conceição & 1 & $2,08 \%$ \\
\hline & Farrapos & 1 & $2,08 \%$ \\
\hline & Floresta & 1 & $2,08 \%$ \\
\hline & IAPI & 1 & $2,08 \%$ \\
\hline & Ilha Pintada & 2 & $4,17 \%$ \\
\hline & Jardim Itu & 1 & $2,08 \%$ \\
\hline \multirow{3}{*}{$\begin{array}{l}\text { Norte/Eixo-Baltazar } \\
\text { (NEB) }\end{array}$} & Costa e Silva & 1 & $2,08 \%$ \\
\hline & Nossa Senhora Aparecida & 1 & $2,08 \%$ \\
\hline & Rubem Berta & 1 & $2,08 \%$ \\
\hline \multirow{4}{*}{$\begin{array}{c}\text { Restinga/Extremo-Sul } \\
\text { (RES) }\end{array}$} & Núcleo Esperança & 2 & $4,17 \%$ \\
\hline & Paulo Viaro & 1 & $2,08 \%$ \\
\hline & Ponta Grossa & 2 & $4,17 \%$ \\
\hline & Restinga & 1 & $2,08 \%$ \\
\hline \multirow{3}{*}{$\begin{array}{l}\text { Lomba do Pinheiro/Partenon } \\
\text { (PLP) }\end{array}$} & Herdeiros & 1 & $2,08 \%$ \\
\hline & Panorama & 2 & $4,17 \%$ \\
\hline & Viçosa & 1 & $2,08 \%$ \\
\hline \multirow[t]{5}{*}{ Centro } & Modelo & 1 & $2,08 \%$ \\
\hline & Santa Marta & 2 & $4,17 \%$ \\
\hline & FASE & 1 & $2,08 \%$ \\
\hline & POA-1 & 1 & $2,08 \%$ \\
\hline & Saúde Indígena & 1 & $2,08 \%$ \\
\hline Não respondeu & & 1 & $2,08 \%$ \\
\hline
\end{tabular}


Tabela 2. Número de consultas utilizadas para a conclusão do tratamento endodôntico

\begin{tabular}{ccc}
\hline Número de consultas & Número de casos concluídos & $\mathbf{\%}$ \\
\hline 1 & 2 & $3,57 \%$ \\
2 & 15 & $26,79 \%$ \\
3 & 17 & $30,36 \%$ \\
4 & 7 & $12,50 \%$ \\
5 & 3 & $5,36 \%$ \\
6 & 3 & $5,36 \%$ \\
7 & 1 & $1,79 \%$ \\
Não respondeu & 08 & $14,29 \%$ \\
\hline Total & 56 & $100,00 \%$ \\
\hline
\end{tabular}

$\mathrm{Na}$ tabela 3 encontram-se as variáveis referentes às condições preconizadas pelo Manual de Especialidades em Saúde $\mathrm{Bucal}^{8}$ no que diz respeito aos encaminhamentos. A tabela 4 descreve as variáveis relativas à ficha de referência dos pacientes encaminhados ao CEO de Endodontia da UFRGS.
O tempo que o paciente teve que aguardar entre a data do encaminhamento da UBS e o atendimento especializado foi inferior a 6 meses em $83,33 \%$ dos casos (40 pacientes), em $10,42 \%$ dos casos o tempo de espera foi entre 6 meses e 1 ano ( 5 pacientes) e $6,25 \%$ dos pacientes não souberam informar o tempo de espera (3 casos).

Tabela 3. Descrição das variáveis referentes às condições preconizadas pelo Manual de Especialidades em Saúde Bucal $(2008)^{8}$ no que diz respeito aos encaminhamentos

\begin{tabular}{cccc}
\hline Variável & Resposta & $\begin{array}{c}\text { Número de } \\
\text { encaminhamentos }\end{array}$ & $\mathbf{\%}$ \\
\hline Indicação de outro elemento dentário a ser & Não & 40 & $83,33 \%$ \\
tratado? & Sim & 6 & $12,50 \%$ \\
Apresenta remoção total do tecido cariado, & Sem resposta & 2 & $4,17 \%$ \\
abertura câmara pulpar, colocação de curativo de & Não & 9 & $18,75 \%$ \\
demora e material restaurador provisório? & Sim & 38 & $79,17 \%$ \\
Apresenta coroa clínica suficiente para colocação & Sem resposta & 1 & $2,08 \%$ \\
dos grampos de isolamento absoluto? & Não & 1 & $2,08 \%$ \\
& Sim & 46 & $95,83 \%$ \\
Apresenta mobilidade acentuada? & Sem resposta & 1 & $2,08 \%$ \\
& Não & 44 & $91,67 \%$ \\
Apresenta adequação do meio bucal com remoção & Sim & 2 & $4,17 \%$ \\
dos focos infecciosos? & Sem resposta & 2 & $4,17 \%$ \\
& Não & 2 & $4,17 \%$ \\
& Sem resposta & 44 & $91,67 \%$ \\
\hline
\end{tabular}


Tabela 4. Descrição das variáveis relacionadas à ficha de referência

\begin{tabular}{cccc}
\hline Variável & Resposta & $\begin{array}{c}\text { Número } \\
\text { de pacientes }\end{array}$ & \% \\
\hline O paciente foi encaminhado através da ficha & Não & 3 & $6,25 \%$ \\
de referência? & Sim & 45 & $93,75 \%$ \\
A ficha de referência contém explicação da & Não & 13 & $27,08 \%$ \\
condição atual do dente? & Sim & 30 & $62,50 \%$ \\
& Sem resposta & 5 & $10,42 \%$ \\
A ficha de referência contém especificado & Não & 4 & $8,33 \%$ \\
corretamente o número do dente? & Sim & 40 & $83,33 \%$ \\
A ficha de referência contém o motivo do & Sem resposta & 4 & $8,33 \%$ \\
encaminhamento? & Não & 7 & $14,58 \%$ \\
& Sim & 37 & $77,08 \%$ \\
A fícha de referência contém assinatura e & Sem resposta & 4 & $8,33 \%$ \\
carimbo do profissional? & Não & 3 & $6,25 \%$ \\
& Sim & 41 & $85,42 \%$ \\
& Sem resposta & 4 & $8,33 \%$ \\
\hline
\end{tabular}

\section{DISCUSSÃO}

Observa-se na atualidade uma grande demanda por tratamento odontológico especializado na rede pública de saúde do Brasil, porém há uma escassez de estudos direcionados aos CEO. A especialidade de Endodontia apresenta grande procura pelos usuários e, portanto, torna-se importante qualificar os serviços ofertados à população.

Estudos mostram uma diferença no padrão de utilização dos serviços de saúde entre homens e mulheres. Segundo dados da Pesquisa Nacional por Amostra de Domicílios (PNAD) realizada pelo Instituto Brasileiro de Geografia e Estatística em 2003, 71,2\% das mulheres e $54,1 \%$ dos homens realizaram consultas médicas naquele ano ${ }^{12}$. Com base no perfil do usuário da atenção secundária em Odontologia no SUS, pacientes do sexo feminino são os que mais utilizam os serviços especializados em odontologia ${ }^{24}$. A presente pesquisa constatou que dos pacientes atendidos no CEO de Endodontia da UFRGS nos anos de 2016 e 2017, 62,5\% eram do sexo feminino, estando de acordo com estudos realizados anteriormente ${ }^{16,18}$. Esses dados indicam que o sexo feminino tem um maior cuidado tanto com sua saúde geral quanto com sua saúde bucal.

Em relação à faixa etária, a maior procura por tratamento foi por adultos entre 31 a 50 anos de idade, seguido por jovens entre 16 a 30 anos. Esse resultado se assemelha ao da pesquisa de Ribeiro et $a .^{20}$, a qual mostrou uma maior procura $\mathrm{e}$ consequentemente necessidade de tratamento endodôntico em pacientes na faixa etária adulta entre 21 e 59 anos. Considerou-se positivo o fato da maior procura dos pacientes adultos e jovens pelos serviços reabilitadores em saúde bucal, uma vez que, por muito tempo, a atenção a esse contingente restringia-se aos atendimentos emergenciais e não conservadores ${ }^{12}$.

A demanda de pacientes idosos foi de apenas 16,67\%. Apesar da ampliação da oferta e da cobertura da assistência odontológica no Brasil, a utilização do serviço por idosos é ainda muito inferior às outras faixas etárias. Há uma demanda acumulada de tratamento e a alta prevalência de perda dentária desacompanhada de reabilitação 
protética, resultado de uma história de modelo de saúde bucal individualista de baixa complexidade e pela ausência de programas específicos para esse grupo $^{17}$.

O sistema municipal de Porto Alegre dispõe de 55 UBS, que, juntamente com 88 Unidades de Saúde da Família (USF) são as principais portas de acesso para a busca de atenção primária em saúde. Os pacientes atendidos no CEO de Endodontia da UFRGS foram encaminhados por 38 diferentes Unidades de Saúde e seis CEO. Os encaminhamentos para os CEO são por proximidade da Unidade de Saúde. Sempre existem três opções mais próximas, porém se existem vagas remanescentes o paciente pode ser encaminhado para outro CEO mais distante ${ }^{18}$.

$\mathrm{Na}$ presente pesquisa o grupo de dentes que mais recebeu tratamento endodôntico foram os primeiros molares (24 dentes, 42,85\%, 12 superiores e 12 inferiores). A erupção destes dentes é precoce e dessa forma são frequentemente confundidos com dentes decíduos, não havendo uma preocupação, por parte dos responsáveis, em mantê-los. Além do mais, sabe-se que na literatura os primeiros molares são os dentes mais acometidos por lesões cariosas, pois a atividade cariogênica inicia-se no seu longo período de erupção, dessa forma ocorre uma maior retenção de placa, prolongando-se esta situação até os dentes entrarem em oclusão, ao redor dos oito anos de idade $^{6}$.

A literatura em Odontologia evidencia a importância do primeiro molar permanente para a mastigação, a fonética e no estabelecimento e integridade da oclusão, assim, verifica-se, sua relevância para o sistema estomatognático. Dessa forma os cirurgiões-dentistas devem estar atentos às necessidades particulares de cada grupo de dentes e do paciente, tais como o tipo de morfologia oclusal, o estágio de erupção do dente, o risco de desenvolver lesões de cárie, o estado motivacional do paciente para estabelecer uma eficiente higienização e consequentemente, o controle da doença cárie e ainda a conscientização dos pais ou responsáveis sobre a importância dos elementos dentários para a saúde bucal ${ }^{13}$.

A comunicação entre atenção primária e secundária é muito importante para promover o cuidado integral do paciente. Assim, o Ministério da Saúde delineou os tratamentos que devem ser ofertados na atenção primária antes do encaminhamento do paciente à atenção secundária $^{2}$. No presente estudo, em $91,67 \%$ dos casos o usuário apresentava correta adequação do meio bucal atendendo às condições preconizadas pelo Manual de Especialidades em Saúde Bucal. Esse resultado é satisfatório, pois reflete a boa integração entre a atenção primária e secundária no município de Porto Alegre, demonstrando que os protocolos estão sendo seguidos pelos profissionais.

Os resultados desse estudo revelaram que a maior parte dos tratamentos endodônticos foram concluídos em 3 ou 2 sessões, somando $66,67 \%$ dos casos. Esse resultado vai ao encontro aos dados encontrados no estudo de Dorr ${ }^{10}$ que avaliou outro CEO do mesmo município, observando que $81,5 \%$ dos tratamentos endodônticos foram concluídos em até três sessões. Já a pesquisa realizada por Saliba et $a .^{23}$, que avaliou um CEO no estado de São Paulo, revelou que foram necessárias em média 4,1 consultas para a finalização dos tratamentos endodônticos. Com base nessas informações fica evidente que os estagiários do CEO de Endodontia da UFRGS apresentam produtividade compatível com profissionais de outros CEO do país, pois a média de consultas para finalização dos tratamentos é semelhante.

$\mathrm{O}$ uso de técnicas e equipamentos contemporâneos em Endodontia, tais como microscópio, localizadores apicais eletrônicos e instrumentos de níquel titânio acionados por motores rotatórios não só aumentaram a taxa de sucesso do tratamento endodôntico, mas também 
encurtaram o tempo necessário para a conclusão do tratamento $^{26}$. Sabe-se que o tratamento endodôntico em sessão única é uma alternativa de procedimento, uma vez que apresenta resultados clínicos satisfatórios semelhantes aos obtidos em múltiplas sessões, tanto no período pós-operatório, quanto em longo prazo ${ }^{11}$. Diante do exposto, fica visível a necessidade de investimentos em novas tecnologias e equipamentos para a especialidade, a fim de promover maior agilidade e redução no número de consultas para a conclusão do tratamento.

O presente estudo revelou que o tempo decorrido entre a data do encaminhamento e o atendimento no CEO de Endodontia da UFRGS foi inferior a 6 meses na maioria dos casos $(83,33 \%)$, apesar desse tempo não ser o ideal, o resultado é semelhante ao encontrado na pesquisa de Persici ${ }^{22}$, na qual se observa que a especialidade odontológica com maior demanda reprimida em março de 2015 no município de Porto Alegre era a Endodontia, com tempo médio de espera para agendamento de 8,3 meses. A especialidade apresenta os maiores tempos de espera no Brasil, com média de 68,3 dias, sendo que há unidades com fila de 315 dias $^{5}$. Ressalta-se que a grande demanda reprimida e a demora para seu agendamento na rede pública podem ocasionar prejuízos importantes para o paciente e para o sistema, visto que se o tratamento endodôntico não for realizado no tempo clínico adequado haverá maior chance de perda do dente e, por conseguinte, a necessidade de outros tratamentos mais invasivos e onerosos, como os consequentes da perda dentária $^{19}$.

De acordo com o estudo de Limão et $a .^{14}$, a especialidade de Endodontia apresentou um tempo de espera relevante, na esfera nacional, regional e local, fato este que pode ter associação com a alta demanda de procedimentos endodônticos em decorrência da não intervenção em estágios iniciais da cárie dentária. $\mathrm{O}$ tempo de espera por atendimento no serviço especializado se deve a fatores, como demanda reprimida, estrutura inexistente, número de profissionais e organização do serviço ${ }^{23}$. Além disso, sabe-se que o absenteísmo é grande e muitas vezes os profissionais acabam ficando ociosos em função das frequentes faltas dos pacientes. Segundo Cayetano et al. ${ }^{7}$, após realizar uma avaliação da Endodontia no serviço público no Brasil, por meio do PMAQ-CEO em 2017, identificaram a Endodontia como a especialidade que apresenta o maior índice de absenteísmo e a maior fila de espera dentre as oferecidas nos $\mathrm{CEO}$ brasileiros. $\mathrm{O}$ absenteísmo, associado ao fato que o serviço estudado funcionava apenas em três turnos por semana pode explicar o pequeno número de casos concluídos (56 casos) no período estudado. A relevância dos dados citados acima reflete no acesso e na qualidade da atenção secundária em odontologia, além de subsidiar informações importantes para futuras ações tanto no nível da gestão quanto nos diversos pontos da rede.

A maior parte dos pacientes $(93,75 \%)$ atendidos no CEO de Endodontia da UFRGS foram encaminhados por meio da ficha de referência. Esse resultado é positivo, pois é um indicador de que o complexo regulador e o protocolo de organização de fluxos de atendimento funcionam de forma adequada em Porto Alegre. Além disso, na Odontologia, a busca por uma prestação de serviços públicos que atendam ao critério de continuidade pautado no princípio da integralidade ancora-se no entendimento e aplicação dos conceitos de referência e contrarreferência, dessa forma um sistema bem regulado de certa maneira contribuiria com a equidade $^{21}$.

A interface ideal contemplaria o acesso à atenção secundária de todos os casos referenciados sem barreiras; sistema de referenciamento para a atenção secundária dos procedimentos não pertinentes à atenção primária; referências 
apropriadas, em tempo hábil e contrarreferência à atenção primária ${ }^{8}$. A organização, pelos gestores da saúde, de um adequado sistema de referência à atenção especializada ainda representa um dos mais importantes desafios a serem enfrentados e evidencia, no cotidiano dos serviços, a busca por uma assistência integral aos cidadãos ${ }^{21}$.

\section{CONCLUSÃO}

A partir da análise do perfil dos usuários e dos atendimentos realizados no CEO de Endodontia da UFRGS, o presente estudo ressalta a importância e relevância de políticas públicas educativas e preventivas no controle da doença cárie, em especial atenção aos molares, pois estes são os dentes mais frequentemente tratados. Os dados aqui apresentados também podem servir de subsídios para o desenvolvimento de estratégias que ampliem a produtividade através de investimentos em novas tecnologias e equipamentos que possam reduzir o número de consultas necessárias para a finalização dos tratamentos endodônticos, proporcionando atendimento para um número maior pacientes e reduzindo o tempo de espera dos pacientes.

\section{ABSTRACT \\ Profile of users and attendances of Endodontics at CEO-UFRGS between 2016 and 2017}

The Dental Specialties Centers (CEO) are a strategy of the National Oral Health Policy to assure the secondary care in Dentistry, constituting referral units for the primary attention. This cross-sectional study analyzed the characteristics of users and attendances in the specialty of Endodontics of the CEO at Universidade Federal do Rio Grande do Sul. A questionnaire was designed, based on the Oral Health Specialties Handout, besides questions that characterized the profile of users, and was applied to the trainees at the CEO at every completed endodontic treatment. The sample included 48 patients, obtained by convenience, in consecutive census sampling, with conclusion of
56 endodontic treatments during the period August 2016 to July 2017. The profile of users was predominantly females $(62.50 \%)$, in the age range 31 to 50 years $(41.67 \%)$ of White ethnicity $64.58 \%)$; the teeth with greatest treatment needs were the molars $(67.86 \%)$; and most cases were in conditions compatible with those advocated by the handout. The treatments were completed in 2 or 3 sessions $(66.67 \%)$, and the waiting time of users for attendance was shorter than 6 months $(83.33 \%)$. The patients were indicated using a referral form $(93.75 \%)$. The study revealed data that may contribute to the development of strategies to broaden the productivity by investments in new Technologies and equipment, aiming to reduce the number of sessions required for finalization of endodontic treatments, providing attendance to a larger number of patients and reducing the waiting time.

Descriptors: Secondary Care. Endodontics. Specialties, Dental.

\section{REFERÊNCIAS}

1. Brasil. Ministério da Saúde. Portaria n ${ }^{\circ} 599$, de 23 de março de 2006. Define a implantação de especialidades odontológicas (CEO) e de laboratórios regionais de próteses dentárias (LRPDs) e estabelece critérios, normas e requisitos para seu credenciamento. Diário Oficial da União. 2006 mar. 24; Seção 1. p. 51.

2. Brasil. Ministério da Saúde. Secretaria de Atenção à Saúde. Departamento de Atenção Básica. Manual de especialidades em saúde bucal. Brasília, DF, 2008. 128 p. (Série A. Normas e manuais técnicos).

3. Brasil. Ministério da Saúde. Portaria Interministerial $\mathrm{n}^{\mathrm{o}} 1.646$, de 5 de agosto de 2014. Institui o componente GraduaCEO, no âmbito da Política Nacional de Saúde Bucal, que irá compor a Rede de Atenção à Saúde (RAS), e dá outras providências. Diário Oficial da União. 2014 ago. 6; Seção 1. p. 46.

4. Bulgareli JV, Faria ET, Ambrosano GMB, Vazquez FL, Cortellazzi KL, Meneghim MC, et al . Informações da atenção secundária em 
Odontologia para avaliação dos modelos de atenção à saúde. Rev Odontol UNESP. 2013; 42(4): 229-36.

5. Carrer FCA, Cayetano MH, Figueiredo N, Padilha WWN. O avaliador externo e o usuário do CEO: narrativa de experiência durante o Ciclo I do PMAQ-CEO. In: Figueiredo N, Goes PSA, Martelli PJL. Os caminhos da saúde bucal no Brasil: um olhar quali e quanti sobre os Centro de Especialidade Odontológicas (CEO) no Brasil. Recife: UFPE; 2016. p. 236-52.

6. Carvalho JC, Ekstrand KR, Thylstrup A. Dental plaque and caries on occlusal surfaces of first permanent molars in relation to stage of eruption. J Dent Res. 1989;68 (5):773-9.

7. Cayetano MH, Gabriel M, Almeida FCS, Araújo ME. Retrato da Endodontia no serviço público no brasil por meio do PMAQ-CEO. Anais. São Paulo: Associação Paulista de Cirurgiões Dentistas, Resumo, 2017.

8. Chaves SCL, Soares FF, Rossi TRA, Cangussu, MCT, Figueiredo ACL, Cruz DN, et al. Características do acesso e utilização de serviços odontológicos em municípios de médio porte. Ciênc Saúde Coletiva. 2012; 17(11): 3115-24.

9. Costa JLBM. Avaliação dos serviços públicos de endodontia nos centros de especialidades odontológicas de Minas Gerais [Tese]. Belo Horizonte: Universidade Federal de Minas Gerais; 2016.

10. Dorr GD, Grecca FS, Giordani JMA. Avaliação dos atendimentos endodônticos em um Centro de Especialidades Odontológicas em Porto Alegre, RS. Rev ABENO. 2016; 16(3):85-9.

11. Hizatugu R, KadoE, Miyasaki E, Okino K, Meneghine GP, Matayoshi A. Endodontia em sessão única: mito ou realidade. $1^{\mathrm{a}}$ ed. São Paulo: Atheneu; 2002.
12. Laroque MB, Fassa ACG, Castilhos ED. Avaliação da atenção secundária em saúde bucal do Centro de Especialidades Odontológicas de Pelotas, Rio Grande do Sul, 2012-2013. Epidemiol Serv Saúde. 2015; 24(3): 421-30.

13. Lima MN, Pinheiro MLS, Cunha LM, Albuquerque MRG, Lopes KS, Carneiro SV. Prevalência da perda dos primeiros molares permanentes em crianças de 8 a 12 anos atendidas na Unicatólica. JOAC. 2017;3(1):16.

14. Limão, NP, Protasio, APL, Machado, LS, Gomes, LB, Valença, AMG. Oferta da assistência odontológica especializada na Atenção Básica do Brasil, Nordeste e Paraíba. REFACS [Internet]. 2017 [Acesso em 10 ago. 2018]; 5(Supl.1):131-140. Disponível em: http://seer.uftm.edu.br/revistaeletronica/index. php/refacs/article/view/1987

15. Machado LX, Toassi RFC, Warmling CM. Gestão da Atenção Primária à Saúde: Práticas de acolhimento em saúde bucal na atenção primária à saúde. In: Bulgarelli $\mathrm{A} F$, Nunes ÂMA, Warmiling CM, Hugo FN, Frichembruder K, Lemos VMA. Redes de Atenção à Saúde: Práticas, experiências e propostas na gestão da Saúde Coletiva. Porto Alegre: Rede Unida; 2016. p. 245-288.

16. Malka VB. Perfil de atendimento do Centro de Especialidades Odontológicas de Endodontia da FO-UFRGS [monografia]. Porto Alegre: Universidade Federal do Rio Grande do Sul; 2015.

17. Nunes CSR, Silva MP, Barcessat ARP. Acesso aos serviços de saúde bucal de adultos e idosos. Est Científica UNIFAP. 2017; 7(3): 918.

18. Peron TB. Perfil do atendimento no centro de especialidades odontológicas de endodontia da FO-UFRGS no primeiro semestre de 2016 
[monografia]. Porto Alegre: Universidade Federal do Rio Grande do Sul; 2016.

19. Persici S. Regulação como ferramenta de gestão: análise da compatibilidade entre oferta e demanda por consultas odontológicas especializadas no município de Porto AlegreRS [monografia]. Porto Alegre: Universidade Federal do Rio Grande do Sul; 2015.

20. Ribeiro ILA, Veloso HHP, Valença AMG, Brasil Júnior O, Lima Neto EA. Avaliação da qualidade de vida e nível de satisfação do usuário da rede de atenção especializada com o tratamento endodôntico no município de João Pessoa, Paraíba, Brasil, 2009. ROBRAC. 2012; 21(59):557-63.

21. Rodrigues L A, Vieira JDM, Leite ICG. Avaliação do fluxo de referência para um centro de especialidades odontológicas implantado em cidade de médio porte na região Sudeste. Cad Saúde Colet. 2013; 21(1): 40-45.

22. Rösing CK, Daudt F. A perspectiva da Atenção Especializada em Periodontia. Porto Alegre, 2016. Apresentação em PowerPoint. [Acesso em 10 ago. 2018]. Disponível em: http://189.28.128.100/dab/docs/portaldab /documentos/mesa iv periodontia ceo.pdf
23. Saliba NA, Nayme JGR, Moimaz SAS, Cecilio LPP, Garbin CAS. Organização da demanda de um Centro de Especialidades Odontológicas. Rev Odontol UNESP. 2013; 42(5): 317-23.

24. Silva HEC, Gottems LBD. Interface entre a Atenção Primária e a Secundária em odontologia no Sistema Único de Saúde: uma revisão sistemática integrativa. Ciênc Saúde Coletiva. 2017; 22(8): 2645-57.

25. Souza GC, Lopes MLDS, Roncalli AG, Medeiros-Junior A, Clara-Costa IC. Referência e contra referência em saúde bucal: regulação do acesso aos centros de especialidades odontológicas. Rev Salud Pública. 2015; 17(3): 416-28.

26. Symanski NC. Terapia Endodôntica: sessão única x sessão múltipla: revisão de literatura [monografia]. Porto Alegre: Universidade Federal do Rio Grande do Sul; 2015.

\section{Correspondência para:}

Simone Bonato Luisi

e-mail: simone.luisi@ufrgs.br

Faculdade de Odontologia da UFRGS

Rua Ramiro Barcelos, 2492

90035-004 - Porto Alegre/RS 